\title{
Prediction of postoperative mortality in elderly patient with hip fractures: a single- centre, retrospective cohort study
}

\author{
Romain Niessen ${ }^{1 *} \mathbb{D}$, Benoit Bihin ${ }^{2}$, Maximilien Gourdin ${ }^{3}$, Jean-Cyr Yombi ${ }^{4}$, Olivier Cornu ${ }^{5}$ and Patrice Forget ${ }^{6}$
}

\begin{abstract}
Background: Elderly patients are at high risk for postoperative complications and increased mortality after hip fracture (HF) surgery due to frailty and co-morbidities. The prediction of postoperative outcome could be used for clinical decision making. A reliable score to predict postoperative mortality after HF surgery in this sub-population remains unavailable.
\end{abstract}

Methods: A single-centre retrospective cohort study was performed in 782 patients who were operated on for HF. Receiver Operating Characteristic (ROC)-curves were used to analyse the performance of gender, age, neutrophil-tolymphocyte ratio (NLR) and C-reactive protein (CRP) at admission $\left(\mathrm{D}_{0}\right)$ as prognostic factors, alone or combined with the PreOperative Score to predict PostOperative Mortality (POSPOM) in univariate and multivariate linear regression models.

Results: No correlation between gender, age, NLR $D_{0}$ or CRP $D_{0}$ and postoperative, intra-hospital mortality was found. The Area Under the ROC-curve (AUC) for age, male gender, NLR and CRP were 0.61 [95\% confidence interval $(\mathrm{Cl})=0.45-0.61], 0.56$ [95\% Cl=0.42-0.56], $0.47[95 \% \mathrm{Cl}=0.29-0.47]$ and $0.49[95 \% \mathrm{Cl}=0.31-0.49]$ respectively. Combination with the POSPOM score did not increase its discriminative capacity as neither age (AUC $=0.69$, $95 \% \mathrm{Cl}=0.54-0.69$ ), gender ( $A \cup C=0.72,95 \% \mathrm{Cl}=0.58-0.72$ ), $\mathrm{NLR} \mathrm{D}_{0}(\mathrm{AUC}=0.71,95 \% \mathrm{Cl}=0.56-0.71$ ), nor the CRP $D_{0}$ (AUC $=0.71,95 \% \mathrm{Cl}=0.58-0.71$ ) improved the POSPOM performance.

Conclusions: Neither age, gender, NLR $D_{0}$ nor CRP $D_{0}$ are suitable parameters to predict postoperative, intrahospital mortality in elderly patients undergoing surgery for HF.

Keywords: Biomarkers, C-reactive protein, Frailty, Hip fractures, Hospital mortality

\section{Background}

Elderly patients are at high risk for postoperative complications and increased mortality after hip fracture (HF) surgery due to frailty and co-morbidities. According to available studies, one-year mortality after HF surgery ranges from 12 to 37\% [1]. The prediction of postoperative outcome could help with clinical decision making. In previous years, several postoperative mortality scores have been developed for this specific purpose in the setting of elective surgeries. However, these scores often

\footnotetext{
* Correspondence: romain.niessen@student.uclouvain.be

'Department of Anesthesiology, Université catholique de Louvain (UCL), CHU

UCL Namur, 1 avenue Dr G Therasse, 5530 Yvoir, Namur, Belgium

Full list of author information is available at the end of the article
}

misinterpret orthogeriatric patients' outcomes and risk of death [2-4].

The PICO model was used to clearly define a clinical question. The population is represented by elderly patients with hip fractures. The intervention aims to identify prognostic factors which improve the accuracy of postoperative mortality prediction in the specific sub-population. Four potential prognostic factors were examined, namely: CRP $D_{0}$, NLR $D_{0}$, age and gender. C-reactive protein (CRP) and the neutrophil-to-lymphocyte ratio (NLR) reflect the inflammatory status of patients during the peri-operative period. Furthermore, the NLR at admission $\left(D_{0}\right)$ and at the fifth day $\left(D_{5}\right)$ have been shown to be associated with postoperative complications [3, 5-7]. We compared predicted mortality to observed mortality. The

(C) The Author(s). 2018 Open Access This article is distributed under the terms of the Creative Commons Attribution 4.0 International License (http://creativecommons.org/licenses/by/4.0/), which permits unrestricted use, distribution, and 
outcome of the study is to improve mortality prediction by analysing if the addition of these variables to the POSPOM score, one of the best validated prognostic tools in the peri-operative period, may increase its discriminative capacity.

\section{Methods}

This study is presented according to the STROBE guidelines (www.strobe-statement.org).

\section{Study design}

Retrospective analysis of a single-centre cohort.

\section{Settings}

Included patients were admitted to the university hospital of St. Luc in Belgium (Cliniques universitaires Saint-Luc) between 2010 and 2016. Study cases were directly recruited after diagnosis of hip fracture in the emergency department or surgical ward. Date of admission, date of discharge, date of intra-hospital death, age, sex, co-morbidities, NLR $D_{0}$, CRP $\mathrm{D}_{0}$ values of patients were recorded. Follow-up was terminated upon hospital discharge or intra-hospital death. Data registration and management were performed in agreement with Belgian law and the Helsinki declaration.

\section{Participants}

After receiving ethical committee approval (Commission d'Ethique Biomédicale Hospitalo-Facultaire - CEBHF) of the Catholic University of Louvain (Chairperson: Prof J-M Maloteaux, n'2010/23DEC/406), the authors were granted a waiver for written informed consent due to the retrospective nature of the study and analysis of anonymised data. The database included a total of 782 patients with a diagnosis of HF. Patients lacking personal data concerning co-morbidities were excluded from the study. All patients included in the study were treated following the same early surgical care protocol (i.e. $81 \%$ operated within the first 24 h). This methodology consisted of obtaining medical clearance from the emergency department as soon after the diagnosis as possible. Patients were then wait-listed and commonly operated the same day. In cases of treatment with anti-vitamin $\mathrm{K}$ medication, coagulation was restored with vitamin K. Patients under anti-platelets treatment were operated on without additional delay, in agreement with the surgeon, under general anaesthesia. When possible, regional anaesthesia/analgesia was proposed and performed, including a fascia iliaca block (single shot). Particular attention was paid to haemodynamic control, with the use of invasive blood pressure monitoring and dynamic variables when indicated and applicable. Postoperative follow-up was performed by an inter-disciplinary medical team consisting of orthopaedic surgeons, anaesthetists, geriatricians, an internal medicine specialist dedicated to peri-operative medicine, and physiotherapists.

\section{Variables}

In previous studies, advanced age and male gender were identified as risk factors in patients with $\operatorname{HF}[3,5]$. Thus, age and gender were chosen as variables with potential discriminative capacity.

Age and gender were registered during pre-operative evaluation. Data on NLR $\mathrm{D}_{0}$ and CRP $\mathrm{D}_{0}$ were taken from the first blood sample obtained from the patient at admission and before surgery. In our clinical practice, blood testing is only realised in presence of an anamnestic or clinical problem, in order to not delay surgery. All blood analyses were performed on venous blood samples and were processed in a blood analyser (Sysmex; TAO Medical Electronics, Kobe, Japan) for full blood count and differential count of leukocytes. The NLR value was obtained by calculating the ratio between registered neutrophils and lymphocytes counts. The CRP value was determined based on a serum sample by a turbidimetry process $\left(\mathrm{UniCe}^{\circ}{ }^{\circ} \mathrm{DxC}\right.$ 800; Beckman Coulter, Pasadena, California, USA) and is expressed in $\mathrm{mg}^{-1}$.

The POSPOM score of each patient was calculated as the sum of the points assigned to each item (age, co-morbidities and type of surgery). With regards to the first variable (age), older patients received higher points. The second variable (co-morbidities) consisted of the total number of points assigned to each of the 17 validated co-morbidities. The third variable (type of surgery) was identical for all patients ("orthopaedic trauma") and therefore each patient received 14 points. Depending on the total number of points, a percentage predicted risk of in-hospital mortality was assigned to each patient [8].

\section{Data collection}

Data collection (Date of admission, date of discharge, date of intra-hospital death, age, sex, co-morbidities, NLR $D_{0}, C R P D_{0}$ values) was performed using systematic, standardised and computerised medical charts issued by the institutional software (Medical Explorer v9, Saint-Luc university Hospital, Brussels, 2009).

Control of potential biases was performed using a prospective listing and a standardisation of the data collection process. The increased weight of the variable "age" was intentional.

The population was divided into two groups: patients discharged from the hospital and patients who died in hospital. Quantitative variables such as the NLR, CRP and age were analysed in the descriptive analysis.

\section{Statistical methods}

Pearson's correlation coefficient was computed to identify potential linear association between $\log (\mathrm{NLR})$ and $\log (\mathrm{CRP})$.

In order to determine the performance of each individual variable (original POSPOM setting, age, gender, 
NLR and CRP) in the prediction of postoperative mortality, we computed the AUC with DeLong confidence intervals. A logistic regression model was then used to combine POSPOM score with the four other variables to determine whether the addition of one of these variables could improve the predictive value of the POSPOM score.

All analyses were performed using R 3.3.2 (R Foundation for Statistical Computing, 2016, Vienna, Austria) and the ggplot 2 and pROC packages.

\section{Results}

\section{Participants}

Of the 782 included patients, 72 were aged under 65 years and 32 had a lack of personal data concerning co-morbidities. Of the 678 patients who were enrolled, 326 had NLR and CRP values at admission. Study flow chart including exclusion criteria is shown in Fig. 1.

\section{Descriptive and outcome data}

The POSPOM-score predicted an average mortality of $13.24 \%$, which is in contrast with the observed intra-hospital mortality of $4.5 \%$ (32 out of 678 patients). In women, the observed intra-hospital mortality was $3.9 \%$ (18 of 470 patients) and $6.3 \%$ in men (14 of 208 patients) (Table 1).

The mean age in surviving patients was $84 \pm 7$ years ( minimum age $=65$ years, maximum age $=105$ years $)$ versus $85 \pm 9$ years in non-survivors. The mean NLR $D_{0}$ in survivors was $8 \pm 6$ and $10 \pm 8$ in non-survivors. The mean CRP $\mathrm{D}_{0}$ was $30.63 \pm 52.71 \mathrm{mg} \mathrm{l}^{-1}$ in survivors vs. $47.19 \pm 73.83$ in non-survivors. The mean POSPOM score was 30 in survivors and 32 in non-survivors, representing a predicted mortality of $13.24 \%$. (Table 2).

\section{Main results}

The calculated correlation coefficients between NLR and CRP showed weak correlation with a Pearson $r$ of 0.216

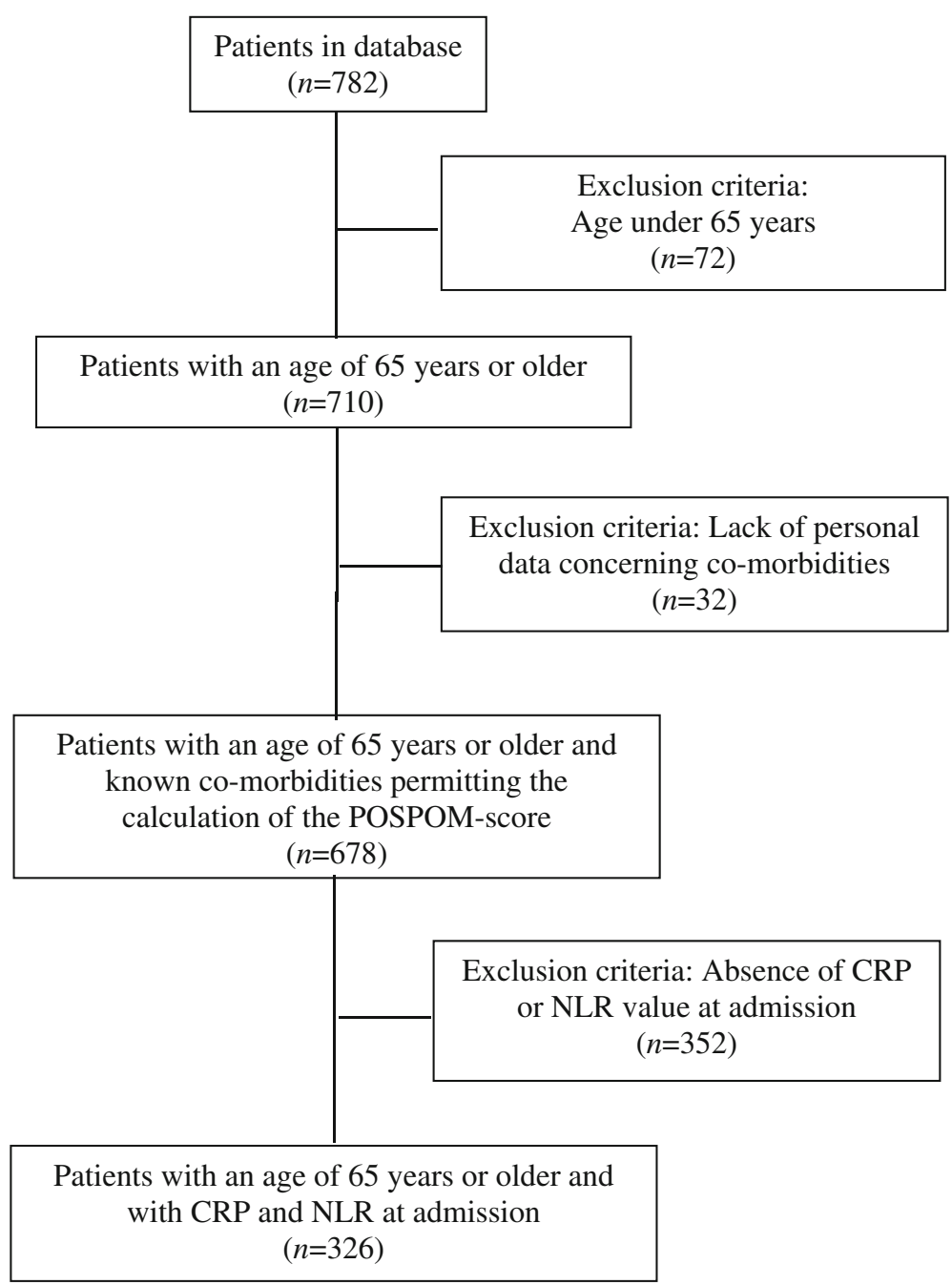

Fig. 1 Patient flow chart diagram 
Table 1 Observed versus predicted mortality in female and male patients

\begin{tabular}{lllll}
\hline Gender & $\begin{array}{l}\text { Number of } \\
\text { patients }(n)\end{array}$ & $\begin{array}{l}\text { Observed Deaths } \\
\text { (patients) }\end{array}$ & $\begin{array}{l}\text { Observed } \\
\text { Mortality (\%) }\end{array}$ & $\begin{array}{l}\text { Predicted } \\
\text { mortality (\%) }\end{array}$ \\
\hline Female & 470 & 18 & 3.6 & 11.61 \\
Male & 208 & 14 & 6.3 & 16.83 \\
All & 678 & 32 & 4.5 & 13.24 \\
\hline
\end{tabular}

[95\% CI $=0.110-0.317]$ and a Spearman r of 0,230 [95\% $\mathrm{CI}=0.159-0.298]$.

The AUC of the complete dataset showed a performance of 0.596 [95\% CI $=0.494-0.596]$ versus an AUC of $0.705[95 \% \mathrm{CI}=0.572-0.705]$ in the restricted dataset with an available NLR and CRP. (Fig. 2).

Subsequently, age, male gender, NLR $\mathrm{D}_{0}$ or CRP $\mathrm{D}_{0}$ were combined to discriminate patients depending on their outcome. AUC for age, male gender, NLR and CRP were 0.608 [95\% CI $=0.453-0.608$ ], 0.560 [CI 95\% $=0.424-0.560$ ], 0.467 [95\% CI $=0.285-0.467]$ and $0.498[95 \% \mathrm{CI}=0.317-0.498]$ respectively (Fig. 3).

Finally, age, male gender, the NLR $\mathrm{D}_{0}$ and CRP $\mathrm{D}_{0}$ were individually combined with the POSPOM (Fig. 4). The predictive performance of the POSPOM is associated with an AUC of 0.705 [95\% CI $=0.572-705$ ] for mortality prediction. Each item was added separately to the POSPOM and the AUC was calculated. Neither age (AUC $=0.693$; 95\% CI $=0.544-$ 0.693), gender ( $\mathrm{AUC}=0.717 ; 95 \% \mathrm{CI}=0.584-0.717$ ), NLR $\mathrm{D}_{0}(\mathrm{AUC}=0.715 ; 95 \% \mathrm{CI}=0.563-0.715)$, nor the CRP $\mathrm{D}_{0}$ (AUC $=0.709 ; 95 \% \mathrm{CI}=0.573-0.709$ ) improved the performance of the POSPOM.

\section{Other analyses}

Despite the incapacity of the four investigated variables to predict postoperative mortality, the authors hypothesised

Table 2 Descriptive analysis showing results of four quantitative variables

\begin{tabular}{|c|c|c|c|c|c|c|c|}
\hline & & Mean & SD & Median & Min & Max & NA \\
\hline \multirow[t]{3}{*}{ Age (years) } & Survivors & 83.649 & 7.390 & 85.0 & 65.0 & 105 & 0 \\
\hline & Non-survivors & 85.312 & 9.146 & 87.5 & 65.0 & 100 & 0 \\
\hline & All & 83.724 & 7.479 & 85.0 & 65.0 & 105 & 0 \\
\hline \multirow[t]{3}{*}{ NLR D $D_{0}$ (ratio) } & Survivors & 8.125 & 5.826 & 6.4 & 0.5 & 45 & 136 \\
\hline & Non-survivors & 9.393 & 5.236 & 7.2 & 2.1 & 27 & 7 \\
\hline & All & 8.183 & 5.796 & 6.6 & 0.5 & 45 & 143 \\
\hline \multirow[t]{3}{*}{ CRP D $D_{0}(\mathrm{mg} / \mathrm{l})$} & Survivors & 30.63 & 52.71 & 7.0 & 1.0 & 421.0 & 328 \\
\hline & Non-survivors & 47.19 & 73.83 & 5.5 & 1.0 & 243.0 & 16 \\
\hline & All & 31.35 & 53.78 & 7.0 & 1.0 & 421.0 & 344 \\
\hline \multirow[t]{3}{*}{ POSPOM (score) } & Survivors & 30.063 & 5.397 & 30.0 & 9.0 & 44 & 0 \\
\hline & Non-survivors & 31.594 & 4.918 & 32.0 & 14.0 & 41 & 0 \\
\hline & All & 30.132 & 5.383 & 30.0 & 9.0 & 44 & 0 \\
\hline
\end{tabular}

SD standard deviation, NA not applicable

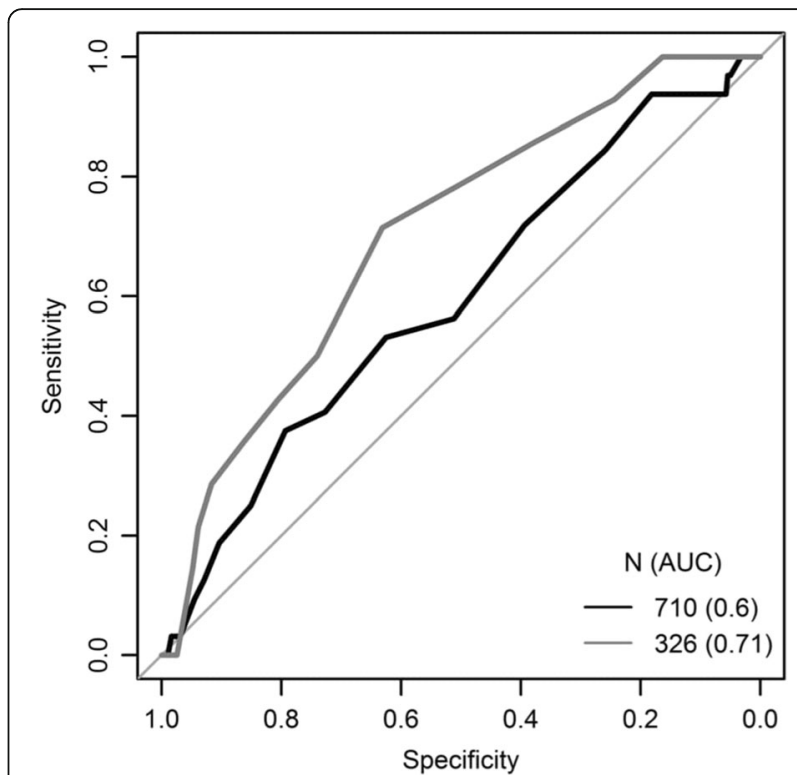

Fig. 2 ROC curves showing complete dataset (after exclusion of patients with an age under 65 years) and restricted dataset of patients with NLR and CRP values. Legend: black line = complete dataset; grey line $=$ restricted dataset

that they may still remain as risk factors. A univariate linear regression model was therefore performed for each variable. The sole statistically significant result was the initial POSPOM setting, with a value of $0.17 \pm 0.07(P=0.019)$. Results of the other univariate regression models for age, male gender, NLR $\mathrm{D}_{0}$ and CRP $\mathrm{D}_{0}$ were $0.053 \pm 0.04(P=0.20)$,

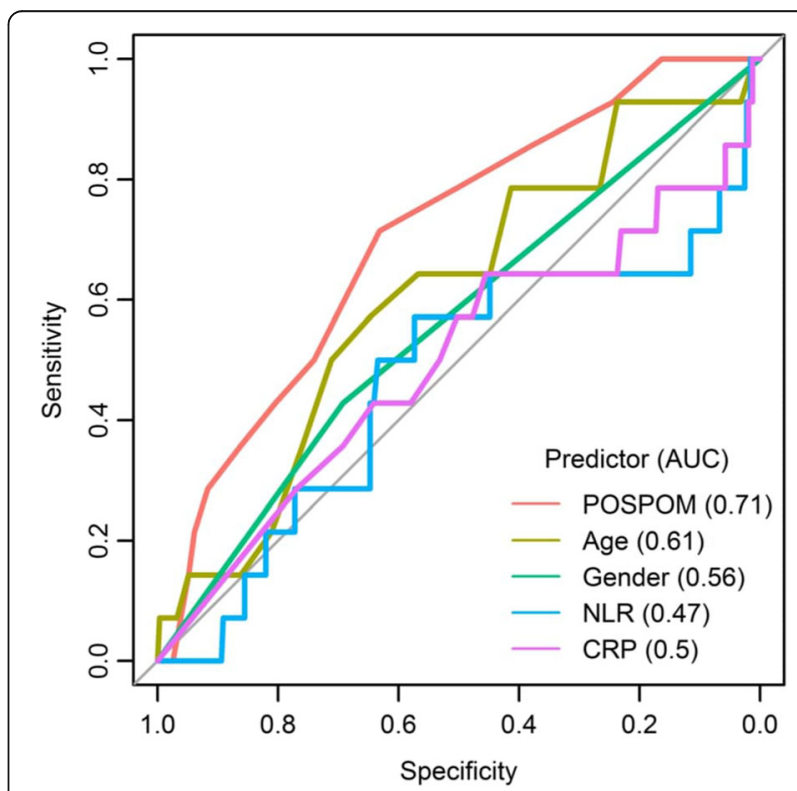

Fig. 3 ROC curves of the restricted dataset and of each variable (univariate linear regression model) 


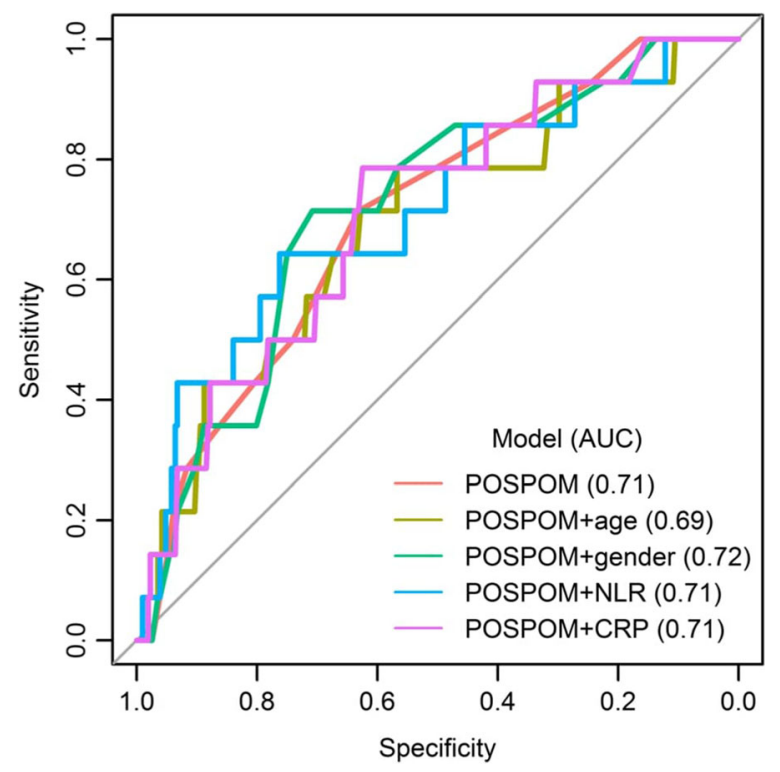

Fig. 4 ROC curves of the combination of restricted dataset with each variable (bivariate linear regression model)

$0.52 \pm 0.55(P=0.34), 0.05 \pm 0.03(P=0.12)$ and $0.01 \pm 0.015$ $(P=0.55)$ respectively. (Table 3$)$.

Finally, a multivariate linear regression model including the POSPOM, age, male gender, NLR $\mathrm{D}_{0}$ and CRP $\mathrm{D}_{0}$ was created. The value for POSPOM was $0.15 \pm 0.07(P=0.046)$, age $0.025 \pm 0.04 \quad(P=0.56)$, male gender $0.25 \pm 0.59$ $(P=0.67)$, NLR $D_{0} 0.058 \pm 0.038(P=0.12)$ and CRP $\mathrm{D}_{0} 0.004 \pm 0.016(P=0.82)$. Data revealed no significant result other than the validation of the discriminative capacity of the POSPOM. (Table 4).

\section{Discussion}

The age, gender, NLR $\mathrm{D}_{0}$ and CRP $\mathrm{D}_{0}$ did not show discriminative capacity in predicting in-hospital mortality after HF. Furthermore, the addition of these variables to the POSPOM did not improve its performance.

Forget and colleagues previously identified age as a risk factor in elderly patients after surgery for $\mathrm{HF}$ [3]. Although age has already been integrated in the original POSPOM, we tested the variable independently in order to give it a higher importance in our scoring system.

The NLR as an inflammatory marker has proven its association with complications and long-term outcome in the

Table 3 Univariate linear regression model

\begin{tabular}{llll}
\hline Variable & Mean & Standard deviation & $P$-value \\
\hline POSPOM & 0.17 & 0.07 & 0.019 \\
Age & 0.053 & 0.04 & 0.20 \\
Male & 0.52 & 0.55 & 0.34 \\
NLR D & 0.05 & 0.03 & 0.12 \\
CRP D & 0.01 & 0.015 & 0.55 \\
\hline
\end{tabular}

Table 4 Multivariate linear regression model

\begin{tabular}{llll}
\hline Variable & Mean & Standard deviation & $P$-value \\
\hline POSPOM & 0.15 & 0.07 & 0.046 \\
POSPOM + Age & 0.025 & 0.04 & 0.56 \\
POSPOM + Male & 0.25 & 0.59 & 0.67 \\
POSPOM + NLR D & 0.058 & 0.038 & 0.12 \\
POSPOM + CRP D & 0.004 & 0.016 & 0.82 \\
\hline
\end{tabular}

setting of gastro-intestinal pathologies treated medically or surgically [6, 9-11]. The NLR has also been associated with complications after major cardiac events or in a variety of cancers [12, 13]. Forget and colleagues developed a four-item score which included the NLR $D_{5}$ after surgery, to predict one-year mortality after surgery for HF [3]. Fisher and colleagues concluded in a recent paper that a high NLR at admission is an independent indicator of fracture risk in orthogeriatric patients and a significant risk factor and moderate predictor for intra-hospital mortality [7].

In our study, all CRP and NLR values taken at admission were included for analysis, simulating real life conditions. The score initially proposed by Forget and colleagues uses the NLR from a blood sample on $D_{5}$, reflecting an inflammatory state [3]. The disadvantage of such a score integrating the NLR at $D_{5}$ is the unavailability of the result on admission.

The calculated POSPOM score of our cohort predicts a mortality of $13.6 \%$. This was higher than the $4.5 \%$ observed in our cohort. This difference may be explained by a multitude of causes.

First, our patients were followed-up using a multidisciplinary approach, the "co-care"-concept. This approach has been shown to reduce mortality compared with standard care [14]. In a meta-analysis and systematic review, Grigoryan and colleagues found that orthogeriatric collaboration was associated with a significant reduction in intra-hospital and long-term mortality, with a relative risk (RR) of 0.60 [95\% CI $=0.43-0.84]$ and $0.83[95 \% \mathrm{CI}=0.74-0.94]$ respectively. Hospital length of stay was reduced in the co-care model, with a standardised mean difference (SMD) of -0.61 [95\% CI $=-0.95,-0.28]$ [15].

Second, in the validation paper published by Le Manach and colleagues, the percentage of major orthopaedic surgery (such as HF) was only $1.76 \%$ of total surgery [8]. Minor surgery was the most represented type of orthopaedic surgery, comprising $19.69 \%$. Consequently, our study population of elderly HF patients was likely under-represented. Third, the optimal timing of surgery for HF in elderly patients still remains unclear [16-18]. Time to surgery was short in our study ( $81 \%$ of patients were operated within $24 \mathrm{~h}$ of admission). As detailed data of the POSPOM population was unavailable, we were unable to determine the significance of this variable.

Recently, several articles have been published showing a lack of accuracy of existing scores. For example, Boddaert 
and colleagues compared pre-operative surgical scores (ASA classification, POSPOM, Nottingham Hip Fracture score) and geriatric scores (Cumulative Illness rating Scale, Charlson comorbidity index) on a dataset of patients hospitalised in a peri-operative geriatric ward: They concluded no superiority in discriminative capacity of specific or geriatric scores in terms of short and long-term postoperative mortality prediction [2]. This result can be explained by the hypothesis that all of the scores are not designed to detect diminished physiological capacity in this frail population and suggest that more attention should be paid to frailty assessment rather than pre-operative characteristics, even in emergency conditions. A rapid, multidisciplinary clinical action plan in a shared ward for this patient sub-set, particularly designed to maintaining autonomy and reduce fall risk, could decrease postoperative mortality.

The intended heterogeneity in this cohort reflects a typical geriatric population and common clinical practice but can be considered as a potential source of bias. Furthermore, the relatively small number of events (32 deaths of 782 patients) limited the power of this study. With regards to the biological markers, the unavailability of the parameters in many patients limits the interpretation of these analyses. Specifically, no exclusion criteria were applied to obtained NLR values despite the fact that patients taking steroid therapy and smokers can show higher neutrophil counts. Patients with malignancies were also not excluded, thereby improving the generalisability of these results, whilst remaining a potential confounding factor. Furthermore, an ongoing infection is often a cause of confusion and fall in geriatric patients, resulting in high CRP $\mathrm{D}_{0}$ and NLR $D_{0}$ values. Also, a malnourished state is frequently observed in elderly patients and is typically associated with lymphopaenia. Finally, the age-related impairment of the immune system may play a role in anomalies in this population [19].

\section{Conclusions}

Age, gender, NLR $\mathrm{D}_{0}$ or CRP $\mathrm{D}_{0}$ have no discriminative capacity to solely predict postoperative mortality after surgery for HF. These variables do not improve the performance of the POSPOM, which remains poor in this population of geriatric patients scheduled for HF surgery.

\footnotetext{
Abbreviations

AUC: Area Under the ROC-curve; Cl: Confidence interval; CRP: C-reactive protein; $D_{0}$ : Day of admission; $D_{5}$ : Fifth day of admission; HF: Hip fracture; NLR: Neutrophil-to-lymphocyte ratio; POSPOM: PreOperative Score to predict PostOperative Mortality; ROC: Receiver Operating Characteristic
}

\section{Acknowledgements}

We would like to thank Laure Nols and Loïc Somerhausen for their technical help. We would like to thank Fritz-Patrick Jahns for his writing assistance.

\section{Funding}

The authors received no specific funding for this work.
Availability of data and materials

Full dataset used and analysed during the current study are available from the corresponding author on request.

\section{Authors' contributions}

All the authors designed the study. RN, JCY and OC collected data. BB analysed data. All the authors contributed to the scientific interpretation. RN drafted the initial text. All the authors revised the manuscript and approved the final version.

\section{Ethics approval and consent to participate}

Ethical approval for this study was obtained by Commission d'Ethique Biomédicale Hospitalo-Facultaire (CEBHF) of the catholic university of Louvain, Chairperson: Prof J-M Maloteaux, n²010/23DEC/406.

\section{Consent for publication}

Not applicable.

\section{Competing interests}

$\mathrm{PF}$ is a member of the Editorial Board (Section Editor).

\section{Publisher's Note}

Springer Nature remains neutral with regard to jurisdictional claims in published maps and institutional affiliations.

\section{Author details}

${ }^{1}$ Department of Anesthesiology, Université catholique de Louvain (UCL), CHU UCL Namur, 1 avenue Dr G Therasse, 5530 Yvoir, Namur, Belgium. ${ }^{2}$ Scientific support unit, Université catholique de Louvain (UCL), CHU UCL Namur, 1 avenue Dr G Therasse, 5530 Yvoir, Namur, Belgium. ${ }^{3}$ Department of Anesthesiology and scientific support unit, Université catholique de Louvain (UCL), CHU UCL Namur, 1 avenue Dr G Therasse, 5530 Yvoir, Namur, Belgium. ${ }^{4}$ Department of Internal and Peri-operative Medicine, Université catholique de Louvain (UCL), Cliniques universitaires Saint-Luc, Brussels, Belgium.

${ }^{5}$ Department of Orthopaedic surgery, Université catholique de Louvain (UCL), Cliniques universitaires Saint-Luc, Brussels, Belgium. ${ }^{6}$ Department of Anesthesiology and Peri-operative Medicine, Vrije Universiteit Brussel (VUB), Universitair Ziekenhuis Brussel (UZ Brussel), Brussels, Belgium.

Received: 20 August 2018 Accepted: 22 November 2018

Published online: 03 December 2018

\section{References}

1. Tajeu GS, Delzell E, Smith W, Arora T, Curtis JR, Saag KG, et al. Death, debility, and destitution following hip fracture. J Gerontol A Biol Sci Med Sci. 2014:69(3):346-53.

2. Boddaert J, Na N, Le Manach Y, Raux M, Cohen-Bittan J, Vallet H, et al. Prediction of postoperative mortality in elderly patients with hip fracture: are specific and geriatric scores better than general scores? Br J Anaesth. 2017;118(6):952-4.

3. Forget $P$, Dillien $P$, Engel $H$, Cornu $O$, De Kock M, Yombi JC. Use of the neutrophil-to-lymphocyte ratio as a component of a score to predict postoperative mortality after surgery for hip fracture in elderly subjects. BMC Res Notes. 2016;9:284.

4. Wang $X$, Zhao BJ, Su Y. Can we predict postoperative complications in elderly Chinese patients with hip fractures using the surgical risk calculator? Clin Interv Aging. 2017:12:1515-20.

5. Forget $P$, Moreau N, Engel H, Cornu O, Boland B, De Kock M, et al. The neutrophil-to-lymphocyte ratio (NLR) after surgery for hip fracture (HF). Arch Gerontol Geriatr. 2015;60(2):366-71.

6. Forget $\mathrm{P}$, Dinant $\mathrm{V}$, De Kock M. Is the neutrophil-to-lymphocyte ratio more correlated than C-reactive protein with postoperative complications after major abdominal surgery? Peer J. 2015;3:e713.

7. Fisher A, Srikusalanukul W, Fisher L, Smith P. The neutrophil to lymphocyte ratio on admission and short-term outcomes in Orthogeriatric patients. Int J Med Sci. 2016;13(8):588-602

8. Le Manach Y, Collins G, Rodseth R, Le Bihan-Benjamin C, Biccard B, Riou B, et al. Preoperative score to predict postoperative mortality (POSPOM): derivation and validation. Anesthesiology. 2016;124(3):570-9. 
9. Cigsar G, Yildirim AC, Anuk T, Guzel H, Gunal E, Gulkan S, et al. Neutrophil to lymphocyte ratio on appendectomy of geriatric and nongeriatric patients. J Investig Surg. 2017;30(5):285-90.

10. Guo J, Chen S, Chen Y, Li S, Xu D. Combination of CRP and NLR: a better predictor of postoperative survival in patients with gastric cancer. Cancer Manag Res. 2018;10:315-21.

11. Khan A, Riaz M, Kelly ME, Khan W, Waldron R, Barry K, et al. Prospective validation of neutrophil-to-lymphocyte ratio as a diagnostic and management adjunct in acute appendicitis. Ir J Med Sci. 2017.

12. Faria SS, Fernandes PC Jr, Silva MJ, Lima VC, Fontes W, Freitas-Junior R, et al. The neutrophil-to-lymphocyte ratio: a narrative review.

Ecancermedicalscience. 2016;10:702.

13. Forget $P$, Bentin C, Machiels JP, Berliere M, Coulie PG, De Kock M. Intraoperative use of ketorolac or diclofenac is associated with improved disease-free survival and overall survival in conservative breast cancer surgery. Br J Anaesth. 2014;113(Suppl 1):i82-7.

14. Fisher AA, Davis MW, Rubenach SE, Sivakumaran S, Smith PN, Budge MM. Outcomes for older patients with hip fractures: the impact of orthopedic and geriatric medicine cocare. J Orthop Trauma. 2006;20(3):172-8 discussion 9-80.

15. Grigoryan KV, Javedan H, Rudolph JL. Orthogeriatric care models and outcomes in hip fracture patients: a systematic review and meta-analysis. J Orthop Trauma. 2014;28(3):e49-55.

16. Klestil T, Roder C, Stotter C, Winkler B, Nehrer S, Lutz M, et al. Immediate versus delayed surgery for hip fractures in the elderly patients: a protocol for a systematic review and meta-analysis. Syst Rev. 2017;6(1):164.

17. Lewis PM, Waddell JP. When is the ideal time to operate on a patient with a fracture of the hip? : a review of the available literature. Bone Joint J. 2016;98-B(12):1573-81.

18. Pincus $D$, Ravi B, Wasserstein D, Huang A, Paterson JM, Nathens AB, et al. Association between wait time and 30-day mortality in adults undergoing hip fracture surgery. JAMA. 2017;318(20):1994-2003.

19. Xia S, Zhang X, Zheng S, Khanabdali R, Kalionis B, Wu J, et al. An update on Inflamm-aging: mechanisms, prevention, and treatment. J Immunol Res. 2016;2016:8426874.

Ready to submit your research? Choose BMC and benefit from:

- fast, convenient online submission

- thorough peer review by experienced researchers in your field

- rapid publication on acceptance

- support for research data, including large and complex data types

- gold Open Access which fosters wider collaboration and increased citations

- maximum visibility for your research: over $100 \mathrm{M}$ website views per year

At $\mathrm{BMC}$, research is always in progress.

Learn more biomedcentral.com/submissions 